\title{
CHandra's Atmospheric Composition Explorer-2 onboard Chandrayaan-2 to study the lunar neutral exosphere
}

\author{
Tirtha Pratim Das ${ }^{1,3, *}$, Smitha V. Thampi ${ }^{2}$, M. B. Dhanya ${ }^{2}$, Neha Naik ${ }^{2}$, \\ P. Sreelatha ${ }^{2}$, P. Pradeepkumar ${ }^{2}$, G. Padma Padmanabhan' ${ }^{2}$ B. Sundar ${ }^{2}$, \\ Dinakar Prasad Vajja ${ }^{2}$, Amarnath Nandi $^{2}$, R. Satheesh Thampi ${ }^{2}$, \\ Vipin K. Yadav' ${ }^{2}$ J. K. Abhishek ${ }^{2}$, Md. Nazeer ${ }^{2}$, P. T. Lali², Rosmy John², \\ A. V. Aliyas ${ }^{2}$, Vijay Kumar Sen ${ }^{2}$, M. Ramprabhu ${ }^{2}$ and A. Ajay Krishna ${ }^{2}$ \\ ${ }^{1}$ Directorate of Technology Development and Innovation, Department of Space, ISRO Headquarters, Antariksh Bhavan, \\ New BEL Road, Bengaluru 560 094, India \\ ${ }^{2}$ Vikram Sarabhai Space Centre, ISRO, Thiruvananthapuram 695 022, India \\ ${ }^{3}$ Formerly at the Space Physics Laboratory, Vikram Sarabhai Space Centre, ISRO, Thiruvananthapuram 695022 , India
}

\begin{abstract}
The CHandra's Atmospheric Composition Explorer-2 (CHACE-2) experiment aboard Chandrayaan-2 orbiter will study in situ, the composition of the lunar neutral exosphere in the mass range 1-300 amu with mass resolution of 0.5 amu. It will address the spatial and temporal variations of the lunar exosphere, and examine water vapour as well as heavier species in it. In this article, results of the major characterization and calibration experiments of CHACE-2 are presented, with an outline of the qualification tests for both the payload and ground segment.
\end{abstract}

Keywords: CHACE-2, Chandrayaan-2, exosphere, mass spectrometer.

\section{Scientific objectives}

\section{Background}

THE Moon has a surface boundary exosphere (SBE), which implies that changes in the composition noticed at any location are directly related to the lunar surface and to any possible release of gases from the lunar interior. The lunar atmospheric composition experiment (LACE) mass spectrometer in Apollo-17, the last of the Apollo series of missions by NASA, USA (1969-1972), could measure only the total surface pressure and a few atmospheric constituents (noble gases) on the nightside of the Moon ${ }^{1,2}$ due to instrument saturation during sunlit conditions. On 14 November 2008, the CHandra's Altitudinal Composition Explorer-1 (CHACE-1) instrument on the Moon impact probe (MIP) of Chandrayaan-1, conducted the first ever in situ measurements of the sunlit lunar neutral exosphere and provided the first altitudinal-

*For correspondence. (e-mail: tp_das@isro.gov.in) latitudinal distribution of tenuous lunar atmospheric composition on the dayside ${ }^{3}$. However, the MIP had been a one-shot mission, i.e. CHACE provided data for about 44 min only with a break up of $20 \mathrm{~min}$ before separation, and 24 min after its separation from the main orbiter Chandrayaan-1 lasting till the time of impact at the lunar south polar region. Nevertheless, these crucial $44 \mathrm{~min}$ data have been extremely useful in furthering our knowledge about the composition of the fragile lunar exosphere. Observations from CHACE in addition to providing the first direct glimpse of the presence of water vapour in the lunar ambience, have also shown, the dominance of neutral atoms/molecules of mass $44 \mathrm{amu}$ in the lunar exosphere ${ }^{3}$, significant quantities of heavier species $(\geq 60 \mathrm{amu})^{4}$, signatures of spatial heterogeneity, north-south asymmetry of radiogenic activities ${ }^{5}$ and provided the 2D distribution of argon, $\mathrm{H}_{2}$, and neon in the lunar exosphere ${ }^{6,7}$, as well as providing the upper limits for helium during extreme conditions ${ }^{8}$. In 2010, the Lunar Crater Observation and Sensing Satellite (LCROSS) impactor of the Lunar Reconnaissance Orbiter (LRO) mission had analysed the ejecta of the impact and detected, water, other volatile compounds, including light hydrocarbons, sulphur-bearing species and carbon dioxide $^{9}$, vindicating the CHACE-1 results.

The Lunar Atmospheric and Dust Environment Explorer $(\mathrm{LADEE})^{10}$ conducted in situ studies of the equatorial (limited to $\pm 23^{\circ}$ lat.) lunar exosphere from December 2013 to April 2014, covering an altitude range of $61 \mathrm{~km}$ down to $4 \mathrm{~km}$. LADEE carried a neutral mass spectrometer (NMS) to study the lunar exosphere in the mass range 2-150 amu. In spite of the wide mass range covered, emphasis had been given only to the noble gases detected by the instrument. LADEE had discovered the presence of $\mathrm{Ne}$ in the lunar exosphere and had studied the variations of $\mathrm{He}, \mathrm{Ne}$ and $\mathrm{Ar}$ in the equatorial latitudes ${ }^{11}$. 
It was also confirmed by the NMS that exospheric helium is controlled by solar wind and by endogenous radiogenic sources. In spite of the significant differences in source strengths, due to the higher lifetime (few months) of $\mathrm{Ne}$ compared to $\mathrm{He}$ (few days), the nightside concentrations of both were shown to be comparable ${ }^{11}$. Localized enhancement of Ar was observed at the Western Maria, coincident with the potassium-rich potassium, rear earth elements and phosphorus (KREEP) regions, vindicating close interaction between the surface and the exosphere, which is characteristic of the surface boundary exospheres ${ }^{11}$. The NMS data have also revealed synodic and semi-annual oscillations of ${ }^{40} \mathrm{Ar}$ of the lunar exosphere $^{12}$. The semi-annual oscillation of $\mathrm{Ar}$ is found to be consistent with condensation loss in the seasonal cold traps at the poles due to the obliquity of the Moon. The results show that the exospheric morphology depends on the energetics of desorption of atoms from soil grains. It also brought out the importance of the surface-exosphere interaction process. The LAMP ultraviolet spectrometer aboard the LRO had detected several lunar species at the day-night terminator, when the surface was dark and the exosphere was sunlit. The upper limits of several lunar species at the terminator regions were also proposed ${ }^{13}$.

The lunar water is another area of scientific interest. While the CHACE experiment aboard the MIP in Chandrayaan-1 reported the first direct signatures of lunar exospheric water vapour in the sunlit side of the Moon ${ }^{14}$, another experiment in the Chandrayaan 1 orbiter, viz. the Moon Mineralogy Mapper experiment inferred the presence of water ice on the lunar surface from the absorption line of the hydroxyl radical at higher latitudes ${ }^{15}$. Recently, results of the detection of water vapour by CHACE in the sunlit lunar exosphere have been vindicated by the LRO observations ${ }^{16}$, which suggest the presence of a monolayer of lunar water thermally adsorbing and desorbing, essentially controlled by the surface temperature.

Despite its scientific importance, the higher latitude regions of the Moon still remain one of the least explored areas. For instance, the Moon's South Pole-Aitken Basin, one of the largest impact structures known within the Solar System, exposes material from deep within the crust and possibly even the upper mantle has been exposed by the impact, and may still preserve molten rocks from the impact itself. The permanently shadowed regions of the Moon show signatures of the presence of hydrogen-bearing compounds ${ }^{17}$, including water. The higher latitude regions of the Moon $\left(70^{\circ}\right.$ and polewards) are the inventories, of the volatiles, where several scientific clues on the origin and evolution of the solar system are believed to be archived.

Therefore, the next logical step is to enable a continuous and quantitative measurement of the lunar exosphere, especially over the high-latitude polar region. The CHACE-2 experiment aboard Chandrayaan-2 orbiter envisages to address the above through a systematic study of the lunar neutral exosphere from $100 \mathrm{~km}$ circular polar orbit.

\section{Science goals of the CHACE-2 experiment}

The scientific objective of CHACE-2 is to characterize the long- and short-term variations of exospheric density and composition, and to understand their causative mechanisms. The scientific questions include especially the diurnal variation and changes in composition during the passage of the Moon through the geomagnetic tail region in each lunation compared to the scenario when the Moon is in the upstream of the Earth's bow shock.

The detection threshold for the CHACE-2 payload is $\sim 10^{2}$ particles $/ \mathrm{cm}^{3}$. The observed densities are expected to be still higher, due to the ram enhancement effect, owing to the relative velocity of the satellite with respect to the lunar exosphere. The ram enhancement will aid the detection of the lunar exospheric species, and this will be accounted for after the reduction of raw spectra to the corrected spectra, with a priori knowledge of the instrument orientation with the satellite velocity vector. The technique of arriving at the corrected spectra from the raw spectra is presented later in the text.

\section{CHACE-2 instrument}

\section{Description}

CHACE-2 is a quadrupole mass spectrometer-based payload in the orbiter segment of the Chandrayaan-2 mission (Figure 1). This instrument, apart from having a quadrupole mass filter, has an in-built Bayard-Alpert (B/A) gauge to measure total pressure. Table 1 presents the specifications of the CHACE-2 payload.

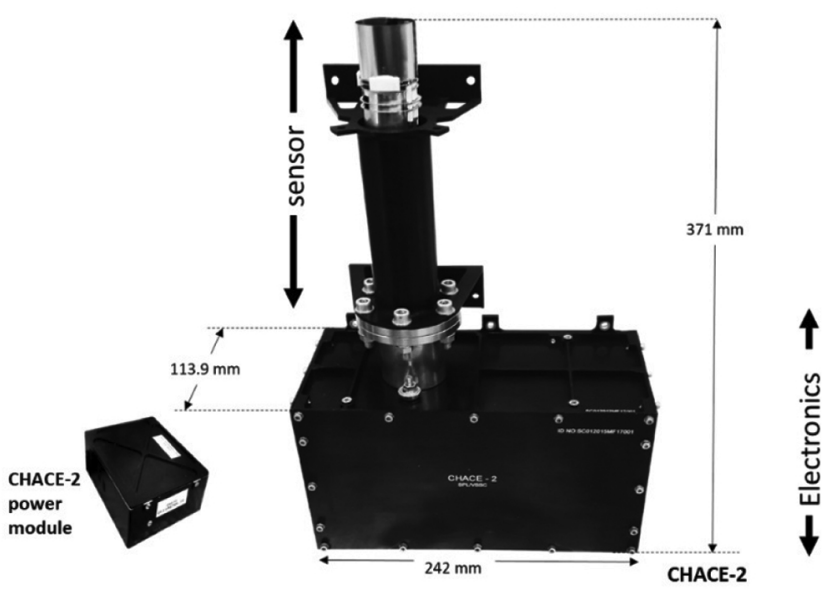

Figure 1. The CHACE-2 (developed by the Space Physics Laboratory, Vikram Sarabhai Space Centre (VSSC), Thiruvananthapuram, payload along with its power module (developed at the U.R. Rao Satellite Centre (URSC), Bengaluru, India). 
Table 1. Specifications of CHACE-2

\begin{tabular}{ll}
\hline Mass range & $\begin{array}{c}\text { 1-300 amu (programmable through telecommand), with } 0.5 \text { amu mass } \\
\text { resolution. }\end{array}$ \\
Ionization technique & $\begin{array}{l}\text { Electron impact ionization with hot cathode filament in an open source } \\
\text { ionizer. }\end{array}$ \\
Quadrupole mass analyser (QMA) for relative abundance measurement \\
Sensors & and Bayard-Alpert (B/A) gauge for total pressure measurement. \\
Detectors & $\begin{array}{l}3.73 \mathrm{~kg} \text { (inclusive of the power module mass of } 0.51 \mathrm{~kg}) . \\
\text { Payload mass }\end{array}$ \\
$\begin{array}{l}33 \mathrm{~W} \text { raw power for nominal operations. } \\
\text { Payload power }\end{array}$ & $242 \mathrm{~mm}(L) \times 113.9 \mathrm{~mm}(B) \times 371 \mathrm{~mm}(H)$. \\
\hline
\end{tabular}

The sensor probe consists of a built-in electron impact ionizer and a Bayard-Alpert (B/A) collector. The ionizer ionizes the ambient neutral atoms and molecules, while the $\mathrm{B} / \mathrm{A}$ collector measures the total pressure. Below the ionizer section, there is a quadrupole mass analyser (QMA), consisting of four quadrupole rods, and a detector assembly. In the ionizer section of CHACE-2, the ambient neutrals are ionized by the energetic (typically $70 \mathrm{eV}$ - programmable through telecommand) electrons emanating from a 'pair' of hot cathode filaments providing redundancy. The quadrupole mass analyser (QMA) system is basically a transmission quadrupole with two pairs of electrically connected, diametrically opposite cylindrical rods and aligned in parallel with the probe axis. Each pair of rods is applied with an RF (frequency $\sim 2.3 \mathrm{MHz}$ ) potential with a superimposed DC potential. The two pairs of rods have an RF phase difference of $180^{\circ}$. The detector assembly comprises a Faraday cup and a channel electron multiplier (CEM). A part of the ambient neutrals gets positively ionized in the ionizer region. The ion optics potential drags the ions formed in the ionizer through an orifice to the entry port of the quadrupole mass filter system. The fraction of the ions formed outside the Faraday cage of the ionizer are collected by the $\mathrm{B} / \mathrm{A}$ collector to constitute a line current, which in turn is calibrated to the total pressure in a $\mathrm{N}_{2}$ dominated ambience.

While at the entrance of the mass analyser all kinds of positively ionized species would be present as a mixture, for a given DC to RF ratio, only the species with a particular charge-to-mass ratio will have stable, oscillatory trajectory to reach the detector and constitute the detector current, while the other species with different charge-tomass ratios would have unstable oscillations with growing amplitude and would not be able to reach the detector. By changing the tuning conditions, mass scanning is accomplished. Thus, the QMA functions like a mass filter. In CHACE-2, the pass band of the mass filter is typically $\sim 0.5 \mathrm{amu}$. Though the Faraday cup and a CEM form a part of the detector system, by default, the pathstable ions impinge on the Faraday cup detector. When the CEM option is chosen, the path-stable ions are repelled after they emerge from the exit aperture of the QMA and are made to impinge on the CEM input. While the Faraday cup has no internal gain, the CEM detector has a large gain in the range $10^{5}-10^{2}$ depending on the bias voltage, mass of the incident ion and to some extent, on the total pressure. The spacecraft interface is based on MIL STD 1553 B; which caters to the telemetry (TM), telecommand (TC) and data interfaces. The CHACE-2 payload is powered by a $\mathrm{DC} / \mathrm{DC}$ converter, which is part of the payload package.

\section{Heritage}

CHACE-2 is a sequel to the CHACE-1 experiment in the Chandrayaan-1/MIP mission ${ }^{18}$ and also draws its heritage from the MENCA payload aboard the Mars Orbiter Mission $(\mathrm{MOM})^{19}$. The major differences between CHACE-1 and CHACE-2 are as follows:

- The mass of the species detectable by CHACE-2 has an extended range of 1-300 amu (programmable), while CHACE-1 had a fixed mass range of 1$100 \mathrm{amu}$.

- Unlike CHACE-1, CHACE-2 is equipped with a provision to track a set of pre-selected species. This comes as an additional feature above the mass scanning capability. However, the mass scan mode is the fundamental mode of operation. Pre-selection of a set of species will be exercised if very high temporal resolution is required at any point of the mission operation.

- Like MENCA aboard MOM, in CHACE-2, there are several payload parameter configurations of operation stored onboard that could be chosen using the telecommand in order to address several scientific questions. On the contrary, CHACE-1 was operated in a single mode, addressing the sunlit lunar exosphere, for a duration of only $44 \mathrm{~min}$.

- CHACE-2 will also be amenable to reloading of the onboard software from ground (using MIL STD I553 Interface).

\section{Characterization and calibration}

CHACE-2 is characterized and optimized with respect to its stabilization time, electron energy for impact ionization of the neutrals, ion optics for focusing the ions to 
the QMA entrance aperture, relative efficiencies of ion transmission through the QMA section, detector response to species of different masses, as well as system noise. After optimization, CHACE-2 is ground-calibrated with residual gases in a vacuum chamber, as well as by introducing different laboratory-grade gases.

Characterization experiments: The closed-loop time constant for a stable emission current in the ion source essentially decides the stabilization time for CHACE-2 from the time it is switched on. The total ion current is bifurcated into two parts; one for mass analysis and the other the $\mathrm{B} / \mathrm{A}$ ionization gauge collector, which in turn is calibrated to total pressure for a nitrogen-rich environment. The B/A collector-to-QMA emission current ratio depends on the potential applied at the ionizer section. For measurement of the relative abundances of the species, the necessary and sufficient condition is that the total emission current has to be stable. However, for estimation of the absolute partial pressures, the condition for the stabilization of the ratio of currents between B/A and QMA also has to be fulfiled. It is observed that the total emission current is stabilized in $\sim 12 \mathrm{sec}$, whereas the ratio of emission current between $\mathrm{B} / \mathrm{A}$ and $\mathrm{QMA}$ takes $\sim 22 \mathrm{~min}$, implying that the payload has to be kept on for at least $\sim 22 \mathrm{~min}$ in order to ensure complete system stabilization.

The detection threshold of a species is limited by the system noise which is characterized with respect to the mass scan rate and CEM bias voltage. Noise data are acquired by turning-off the filament and acquiring the detector output current at the end of the signal conditioning chain. The frequency distribution of noise data at different mass scan rates are plotted in Figure 2 and Gaussian distributions are fitted. It is observed that the system noise has a Gaussian behaviour with mean $\sim 0$ and standard deviation depending on the mass scan rate. The noise floor is chosen as the $3 \sigma$ level.

Figure 3 presents the variation of noise floor with mass scan rate. It is observed that for scan rates at or below 48 points/s, the noise floor remains $\sim 10^{-14} \mathrm{~A}$. The overall threshold of CHACE-2 has been experimentally determined to be $5 \times 10^{-15}$ Torr $\left(\mathrm{N}_{2}\right.$ equivalent partial pressure). For different dominant gases, the threshold would be proportional to the ratio of the electron ionization cross-section of $\mathrm{N}_{2}$ and the dominant species.

The other important set of characterization is the relative transmission efficiencies (quadrupole mass discrimination (QMD)) of ions through QMA, and the relative detection efficiency of the CEM detector.

In the QMD effect, due to the fringing electric fields at the termination of the QMA rods, transmission efficiency of heavier ions is less than the lighter ions. This results into over estimation of the relative abundance of the lighter species with respect to the heavier ones. The relative transmission efficiencies of different ions are characte- rized for different masses. The QMD factors are treated as a set of multiplicative calibration constants in order to compensate for the QMD effect. The QMD factor profile for CHACE-2 is computed by studying the fragmentation pattern at $70 \mathrm{eV}$ electron impact ionization energy and/or the second-to-first ionization peak ratios from the signatures of ${ }^{4} \mathrm{He},{ }^{40} \mathrm{Ar}, \mathrm{N}_{2}$ and $\mathrm{Xe}$ gases, as well as the residual gases in the chamber, and comparing the same with their respective handbook values.

Figure 4 suggests that the QMD effects for CHACE-2 are prominent at masses greater than $80 \mathrm{amu}$. The profile was further verified against the fractionation pattern of the reference material compound perfluorotributylamine (PFTBA; which yields mass peaks at amu 31, 69 and $100 \mathrm{amu}$ in the range $1-100 \mathrm{amu})^{20}$.

Figure 5 shows the gain of the CEM detector as a function of ion mass at different bias voltages. The electron multiplier gain (EMG) profile is characterized to study

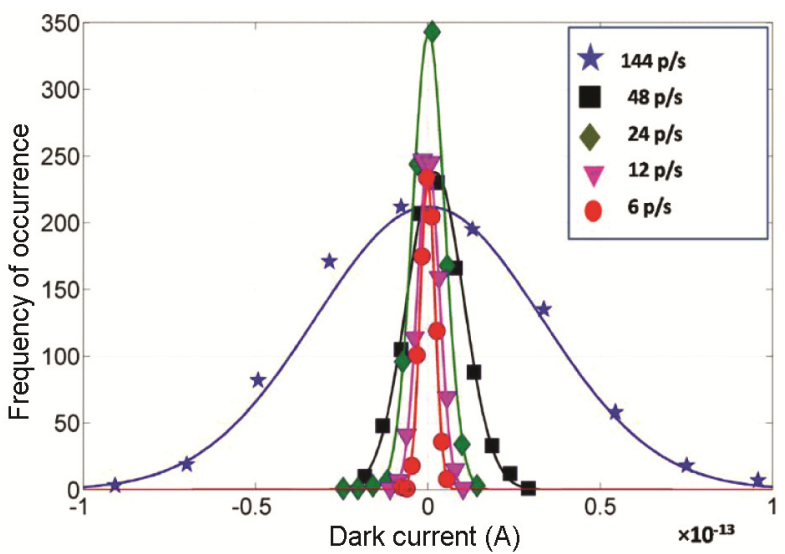

Figure 2. Frequency distribution of system noise of CHACE-2 at different mass scan rates. It is seen to follow Gaussian distribution with mean at $0 \mathrm{~A}$ and standard deviation depending on the mass scan rate.

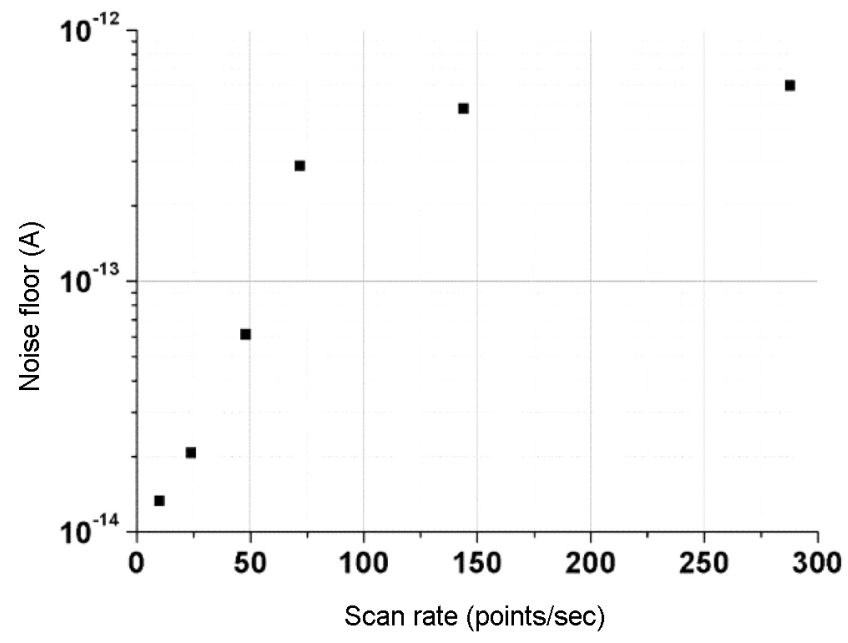

Figure 3. Variation of noise floor with mass scan rate. At slower mass scan rates, the noise averages out resulting in better signal-tonoise ratio (SNR), but at the cost of temporal resolution. 


\section{RESEARCH ARTICLES}

the variation of gain of the CEM detector with the ion mass. The results indicate that the CEM detector has better response to the low mass-to-charge ratio ions. The EMG profile is obtained by comparing the mass spectra acquired with the Faraday cup and the CEM detector. The EMG values are treated as divisive calibration factors in order to account for the lesser sensitivity of the CEM detector to the heavier ions.

The QMD and EMG calibration factors are used while reducing the raw mass spectra for the relative abundances of the detected species. Once corrected for the relative abundances, the spectra are normalized with respect to the total pressure. The calibration of the $\mathrm{B} / \mathrm{A}$ gauge will be described later in the text.

The CEM bias voltage is optimized for maximizing the signal-to-noise ratio (SNR), by making a trade-off

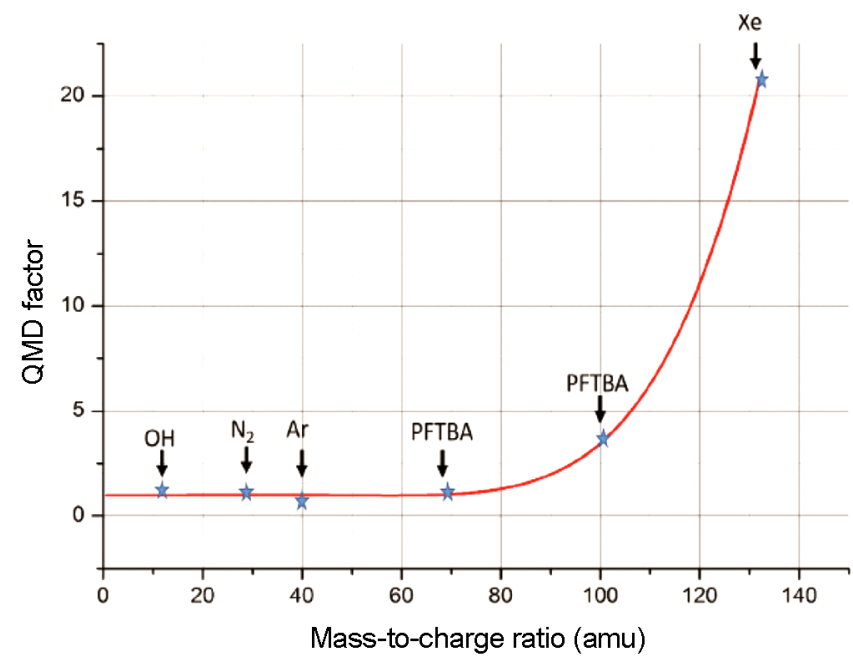

Figure 4. Quadrupole mass discrimination (QMD) factor as a function of ion mass, computed from the signatures of $\mathrm{H}_{2} \mathrm{O}, \mathrm{N}_{2}$, Ar, Xe and confirmed with the PFTBA signature.

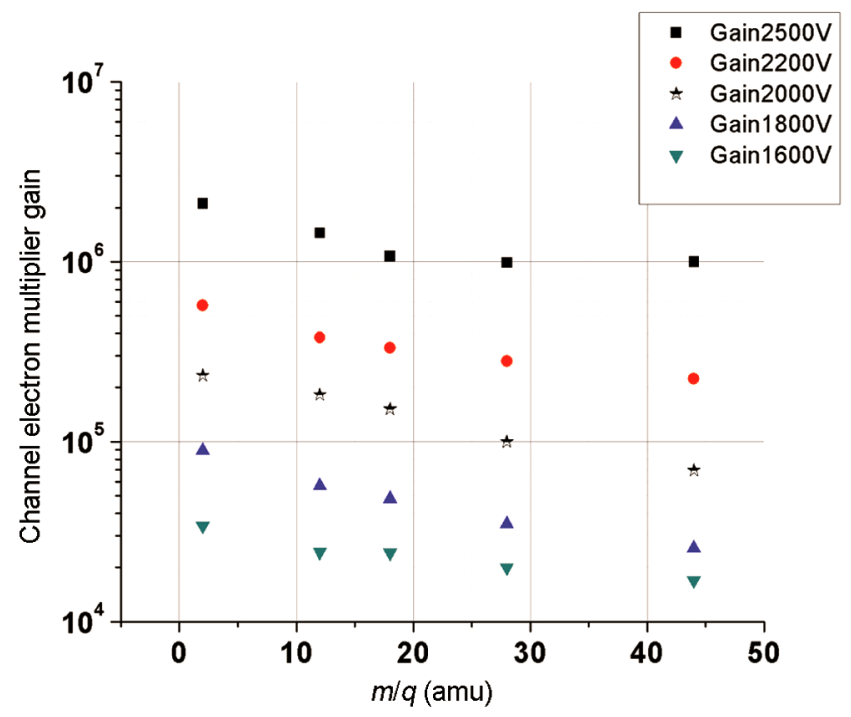

Figure 5. Variation of electron multiplier gain with mass-to-charge ratio of the ions. between system gain and shot noise. In Figure 6, the variation of SNR of 28 amu with respect to the CEM bias voltage is plotted. It is observed that the CEM bias optimizes at $1800 \mathrm{~V}$. However, in CHACE-2, there are options to change the CEM bias voltage through telecommand, for ramp up/down from the optimum level, as well as to cope with any drift of optimum bias voltage during the operational phase of the mission.

The relation between detector current for a given abundance of species and mass resolution has also been studied. Higher mass resolution would reduce the throughput of the quadrupole mass filter. The mass spectra of the residual gases were acquired at a stabilized pressure of $5 \times 10^{-7}$ Torr at different mass resolutions of $\mathrm{m} / \Delta \mathrm{m}=$ 112,58 and 16 respectively, where $m=28$ amu and $\Delta m$ is the peak width at $10 \%$ of the peak height. It is observed that for a variation of mass resolution from 112 to 16 (corresponding variation of $\Delta m$ is from 0.25 to $1.77 \mathrm{amu}$ ), the throughput increased by a factor of $\sim 40$.

Total pressure calibration: Although the static pressure expected in the lunar exosphere is in the range $10^{-10}$ $10^{-12}$ Torr, it is expected that due to the large velocity of the mass spectrometer with respect to the medium and the consequent ram pressure, the total pressure will be measured in the range $10^{-6}-10^{-8}$ Torr. The static pressure at the native lunar environment will be calculated after taking into consideration the dynamic pressure effects. Nonetheless, the total pressure calibration is done by studying the variation of the B/A gauge collector current against the total pressure read by a reference gauge in the pressure range $10^{-6}-10^{-8}$ Torr (Figure 7).

Response of an identical $\mathrm{B} / \mathrm{A}$ gauge studied at static pressures in the range $10^{-7}-10^{-11}$ Torr, revealed that till $3 \times 10^{-9}$ Torr it remained linear, while at lower pressures the $\mathrm{B} / \mathrm{A}$ gauge current and $\mathrm{X}$-ray photocurrent due to high-energy electrons impinging on the $\mathrm{B} / \mathrm{A}$ gauge collector become comparable. It is to be noted that in the dynamic environment, the CHACE-2 B/A gauge would operate in the linear regime. Further, the calibration constant for converting current into number density has been experimentally determined to be $\mathrm{K}=3.54 \times 10^{-22} \mathrm{~m}^{3}$.

\section{Data analysis procedure}

In the mass sweep mode, data contain raw mass spectra comprising detector current and total pressure in a tabular form. Typically, nine consecutive data points constitute a mass bin. That is, the first to the ninth data point represent mass number 1 , 10th to the 18 th data point represent mass number 2 , and so on. In case a signal is detected for a given mass, the data points in the corresponding bin exhibit the rising and trailing edges of the peak. For a symmetrical peak, the data point in the middle of the mass bin has the maximum value of detector 
current. A peak detection algorithm (PDA) is used to detect whether there is a valid peak in a given mass bin $^{8}$.

As the first step in data analysis, PDA is used to detect any possible peak in each mass bin of a raw spectrum. If a peak is detected in a mass bin (say, $j$ ), the value of its amplitude (say, $p_{j}$ ) is returned by the algorithm by fitting a second-degree polynomial on the corresponding data points. Otherwise, the value returned is zero $\left(p_{j}=0\right)$. Thus, for a raw mass spectrum spanning the mass range 1-300 amu, there are 300 values (say, $p_{1}, p_{2}, \ldots, p_{300}$ ) returned by the PDA, corresponding to the respective peak amplitudes. The relative amplitudes of $p_{1}, p_{2}, \ldots, p_{300}$ need to be multiplied with the respective QMD calibration factors $\left(q_{1}, q_{2}, \ldots, q_{300}\right)$ and divided by the respective

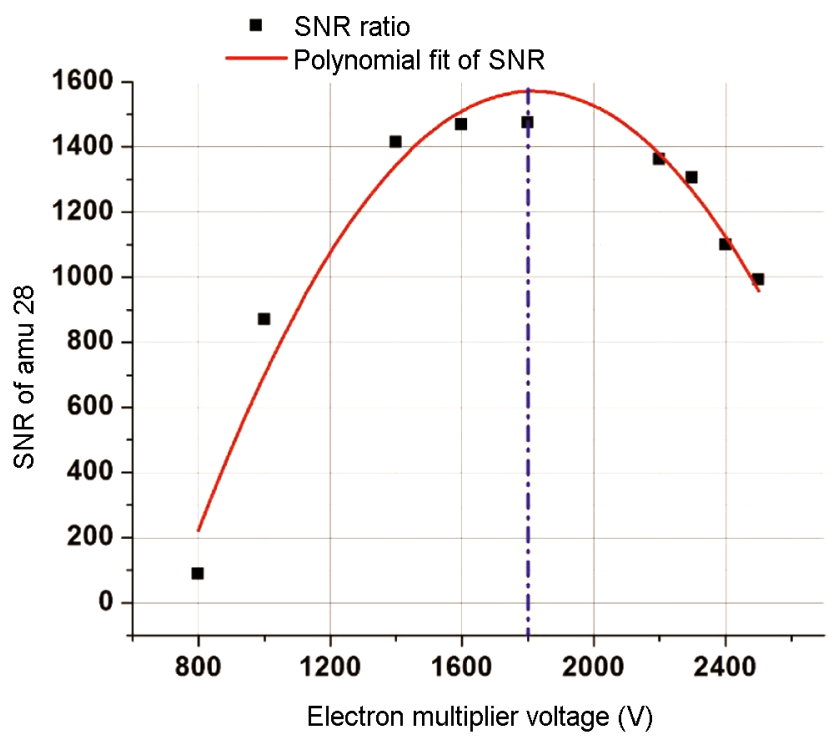

Figure 6. Variation of SNR with CEM bias voltage at a total pressure of $3 \times 10^{-8}$ Torr.

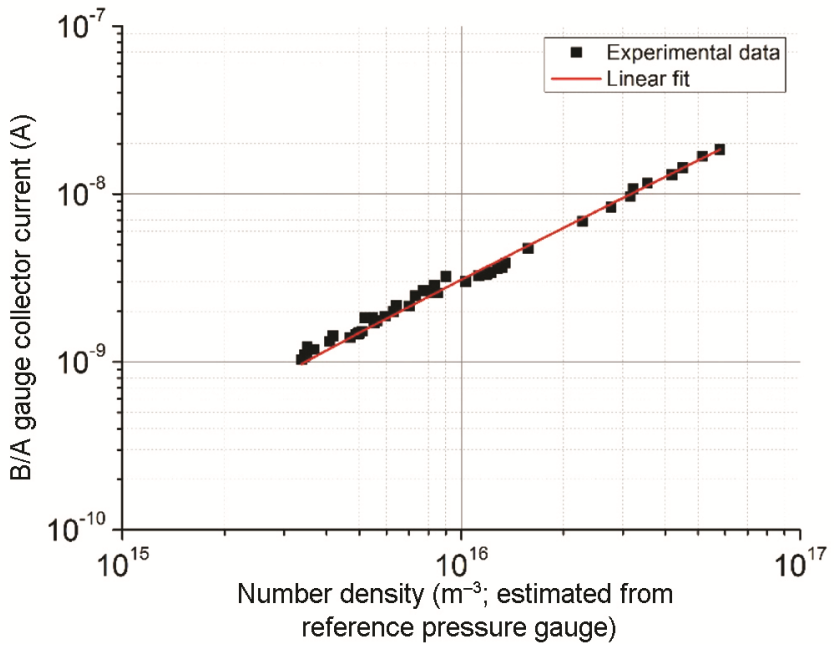

Figure 7. Variation of the B/A gauge collector current $\left(I_{C}\right)$ with number density $(n)$. The variation is linear in the pressure range $10^{-6}$ to $10^{-8}$ Torr, with a slope of $3.19 \times 10^{-25} \mathrm{~A} \mathrm{~m}^{3}$. A number density of $10^{15} \mathrm{~m}^{-3}$ corresponds to a pressure of $3.1 \times 10^{-8}$ Torr at $300 \mathrm{~K}$ temperature using ideal gas law.
EMG calibration factors $\left(g_{1}, g_{2}, \ldots, g_{300}\right)$, so as to read the correct relative abundances of the species. Each raw spectrum has a corresponding total pressure $\left(P_{T}\right)$. The dominant species is identified from the relative abundances of the mass spectra. The relative sensitivity factor for ionization of the dominant species, say $k_{r}$, is used to arrive at the corrected total pressure $P_{T c}$, given by $P_{T c}=P_{T} / k_{r}$.

Next, the sum of the corrected relative abundances are normalized to the total pressure indicated by the $\mathrm{B} / \mathrm{A}$ gauge.

Figure 8 presents the procedure described above as a flowchart.

\section{Instrument qualification}

CHACE-2 has undergone all the mandatory tests before integration with the spacecraft. Both the mechanical and electrical parameters are verified during the test and evaluation process. The tests are divided into three main categories, viz. (i) initial standard room condition tests (ISRC); (2) environmental tests, and (iii) final standard room condition (FSRC) tests.

Further, MIL-STD 1553 interface validation tests are carried out to verify that the design of the onboard system of the payload meets the necessary requirements. These include electrical and protocol tests. The tests are conducted on both the main and redundant buses. EEPROM checks are conducted to ensure proper write-ability and read-ability of EEPROM in the onboard system and also to ensure that the write protection features of EEPROM are effective.

The environmental tests on the payload are conducted according to the environmental test-level specifications (ETLS) defined by the mission. They include EMI/EMC, thermal soak, vibration and thermo-vacuum cycling.

FSRC tests are performed in a controlled environment after all the environmental tests. All tests performed during the ISRC are repeated during FSRC. The results of ISRC and FSRC are compared to check for data integrity of the payload.

Apart from the detailed test and evaluation of the payload, a detailed failure mode effect and critical analysis (FMECA) of the onboard system of the CHACE-2 payload has also been carried out. Failure modes of all interfaces with respect to the orbiter have been analysed to ensure that there is no propagative effect.

\section{CHACE-2 ground segment}

Once the science data from the different payloads on the spacecraft are received at the ground station, Indian Deep Space Network (IDSN), the CHACE-2 data are separated out and a raw data file is formed at the Indian Space Science Data Centre (ISSDC). These data are fed as input 


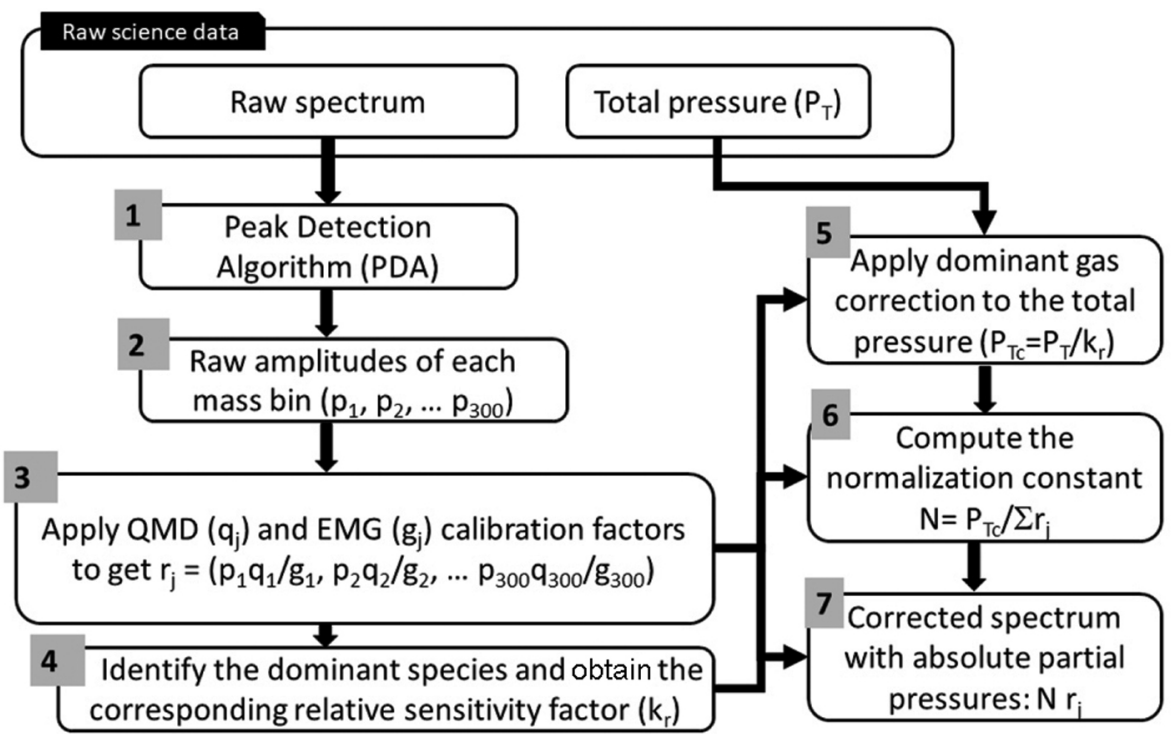

Figure 8. Flowchart for arriving at the corrected spectrum from the raw spectrum.

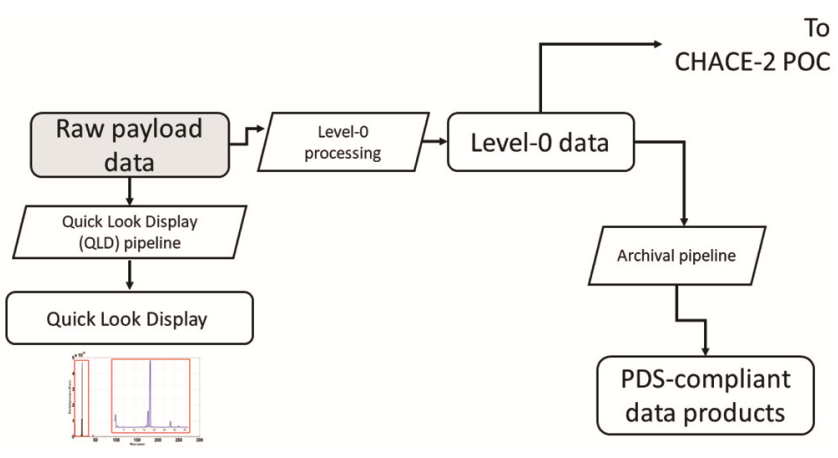

Figure 9. Schematic of CHACE-2 data flow at ground and the different levels of data processing. The level-0 data, once plotted, represent the raw spectra and the B/A gauge collector current. These subsequently have to be corrected using suitable calibration constant/factors, as discussed in the text.

to the CHACE-2 quick look display (QLD) software pipeline, which processes the raw files and generates plots of the relevant science and housekeeping (HK) parameters in an automated manner for quick assessment of instrument health and performance. After the level-0 processing at ISSDC, the science and ancillary data files are fed to the archival pipeline, which generates the science data products according to the planetary data system standards. The level-0 data files are also made available to the CHACE-2 payload operation centre at Space Physics Laboratory, Vikram Sarabhai Space Centre for detailed analysis. Figure 9 is a schematic of the overall data flow and the different levels of data processing for CHACE-2.

\section{Payload Operation Centre}

The CHACE-2 Payload Operation Centre (POC) in SPL will regularly receive the science and $\mathrm{HK}$ data of
CHACE-2 from ISSDC through a network. In addition to the offline processing of HK data, POC will monitor the real-time $\mathrm{HK}$ parameters of $\mathrm{CHACE}-2$ as well. The science data received at POC will be processed immediately to generate the quick-look display plots. The interface from POC to ISSDC is through the National Knowledge Network (NKN). The POC is equipped with storage systems and workstations for storage and analysis of data.

\section{Summary}

The CHACE-2 payload aboard Chandrayaan-2 orbiter is an open-source, quadrupole-based mass spectrometer and can measure both surface-reactive and inert neutral species along the spacecraft track, and is intended to study the composition, structure and variability of the tenuous lunar exosphere. This experiment is expected to provide new insights about the lunar exosphere, including the sources, sinks and their variability, especially pertaining to the polar region. Many 'first-of-its-kind' results are expected from CHACE-2.

1. Stern, S. A., The lunar atmosphere: history, status, current problems, and context. Rev. Geophys., 1999, 37, 453-491.

2. Hoffman, J. H., Hodges Jr, R. R., Johnson, F. S. and Evans, D. E., Lunar atmospheric composition: results from Apollo 17. In Lunar and Planetary Science Conference Proceedings, 1973, vol. 4, p. 2875.

3. Sridharan, R., Ahmed, S. M., Das, T. P., Sreelatha, P., Pradeepkumar, P., Naik, N. and Supriya, G., The sunlit lunar atmosphere: a comprehensive study by CHACE on the moon impact probe of Chandrayaan-1. Planet. Space Sci., 2010, 58, 1567-1577.

4. Sridharan, R., Das, T. P., Ahmed, S. M. and Bhardwaj, A., Indicators for localized regions of heavier species in the lunar surface from CHACE on Chandrayaan-1. Curr. Sci., 2013, 105(11), 14701472 . 
5. Sridharan, R., Das, T. P., Ahmed, S. M., Supriya, G., Bhardwaj, A. and Kamalakar, J. A., Spatial heterogeneity in the radiogenic activity of the lunar interior: Inferences from CHACE and LLRI on Chandrayaan-1. Adv. Space Res., 2013, 51, 168-178.

6. Thampi, S. V., Sridharan, R., Das, T. P., Ahmed, S. M., Kamalakar, J. A. and Bhardwaj, A., The spatial distribution of molecular hydrogen in the lunar atmosphere-new results. Planet. Space Sci., 2015, 106, 142-147.

7. Tirtha Pratim Das, Thampi, S. V., Bhardwaj, A., Ahmed, S. M. and Sridharan, R., Observation of $\mathrm{Ne}$ at mid and high latitudes in the sunlit lunar exosphere: results from CHACE aboard MIP/ Chandrayaan-1. Icarus, 2016, 272, 206-211.

8. Tirtha Pratim Das, Smitha V. Thampi, Dhanya, M. B., Anil Bhardwaj, Ahmed, S. M. and Sridharan, R., Upper limit of helium-4 in the sunlit lunar exosphere during magnetotail passage under low solar wind condition: result from CHACE aboard MIP in Chandrayaan-1. Icarus, 2017, 297, 189-194.

9. Colaprete, A. et al., Detection of water in the LCROSS ejecta plume. Science, 2010, 330(6003), 463-468.

10. Elphic, R. C. et al. The Lunar Atmosphere and Dust Environment Explorer Mission. Space Sci. Rev., 2014, 185, 3-25.

11. Benna, M., Mahaffy, P. R., Halekas, J. S., Elphic, R. C. and Delory, G. T., Variability of helium, neon, and argon in the lunar exosphere as observed by the LADEE NMS instrument. Geophys. Res. Lett., 2015, 42, 3723-3729.

12. Hodges, R. R. and Mahaffy, P. R., Synodic and semiannual oscillations of argon-40 in the lunar exosphere. Geophys. Res. Lett., 2016, 43, 22-27.

13. Cook, J. C., Stern, S. A., Feldman, P. D., Gladstone, G. R., Retherford, K. D. and Tsang, C. C. C., New upper limits on numerous atmospheric species in the native lunar atmosphere. Icarus, 2013, 225, 681-687.

14. Sridharan, R., Ahmed, S. M., Das, T. P., Sreelatha, P., Pradeepkumar, P., Naik, N. and Supriya, G., 'Direct' evidence for water $\left(\mathrm{H}_{2} \mathrm{O}\right)$ in the sunlit lunar ambience from CHACE on MIP of Chandrayaan I. Planet. Space Sci., 2010, 58, 947-950.

15. Pieters, C. M. et al., Character and spatial distribution of $\mathrm{OH} / \mathrm{H}_{2} \mathrm{O}$ on the surface of the moon seen by M3 on Chandrayaan-1. Science, 2009, 326, 568.

16. Amanda, R. H. et al., Diurnally-migrating lunar water: evidence from ultraviolet data. Geophys. Rev. Lett., 2019; doi: 10.1029/2018GL081821.

17. Lawrence, D., Miller, R., Ozimek, M., Peplowski, P. and Scott, C., High-resolution mapping of lunar polar hydrogen with a lowresource orbital mission. Acta Astronaut., 2015, 115, 452-462.

18. Kumar, A. et al., The moon impact probe on Chandrayaan-I. Curr. Sci., 2009, 96(4), 540-543.
19. Bhardwaj, A. et al., MENCA experiment aboard India's mars orbiter mission. Curr. Sci., 2015, 109(6), 1106-1113; doi:10.18520/v109/i6/1106-1113.

20. Chapman, J. R., Practical Organic Mass Spectrometry, John Wiley, Chichester, UK, 1995, 2nd edn.

ACKNOWLEDGEMENTS. This work is supported by the Department of Space (DOS), Indian Space Research Organization (ISRO), Bengaluru, Government of India. The CHACE-2 team thanks to Dr K. Sivan (Chairman, ISRO), Bengaluru for active guidance provided throughout the process and stringent review at the system level. T.P.D. thanks Shri R. Umamaheshwaran (Director, DTDI/Scientific Secretary, ISRO), for support. The CHACE-2 team also thanks Shri S. Somanath (Director, VSSC, Thiruvananthapuram); Shri V. Kishornath (Associate Director, VSSC), Dr Radhika Ramachandran (Director, SPL-VSSC); the Payload Review and Clearance Board (PRCB), and various other review committees for stringent and meticulous reviews at different phases of the technical development and qualification. We acknowledged close association of the Quality Assurance, Quality Control, Software Quality Assurance and review teams, Test and Evaluation, Configuration Control teams and their valuable suggestions and guidance throughout. The CHACE-2 team also acknowledges the persistent support of the personnel of the Environmental Test Facility, Vibration Laboratory and EMI/EMC Laboratory. We thank the teams at URSC, Bengaluru for help in developing the customized power module for CHACE-2, as well as for the integrated spacecraft-level tests; IRSPMO team for providing electronic components; ISSDC and ISTRAC teams for the ground segment support. The contributions of the Avionics, Aeronautics, System Reliability, Structures, Mechanisms and Vehicle Integration Testing, Materials and Mechanical Entity and other entities of VSSC are also acknowledged. The CHACE-2 team thanks Dr Anil Bhardwaj (Director, PRL, Ahmedabad), Shri A. S. Kiran Kumar (former Chairman, ISRO), Prof R. Sridharan (NASI Honorary Scientist, Physical Research Laboratory (PRL), Ahmedabad) and Dr T. K. Alex (Honorary distinguished professor, ISRO) for valuable scientific discussions and timely advice. We also thank the Chandrayaan-2 project, Ms M. Vanitha (Project Director, Chandrayaan-2), Shri G. Nagesh (Program Director) and Dr P. Sreekumar (Director, Space Science Programme Office), ISRO Headquarters, Bengaluru for their persistent support.

Received and accepted 27 August 2019

doi: $10.18520 / \mathrm{cs} / \mathrm{v} 118 / \mathrm{i} 2 / 202-209$ 